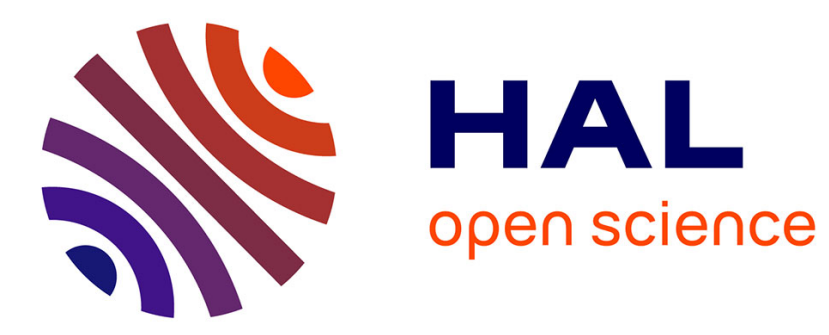

\title{
Study of First-Order Thermal SIGMA-DELTA Architecture for Convective Accelerometers
}

Olivier Leman, Frédérick Mailly, Laurent Latorre, Pascal Nouet

\section{To cite this version:}

Olivier Leman, Frédérick Mailly, Laurent Latorre, Pascal Nouet. Study of First-Order Thermal SIGMA-DELTA Architecture for Convective Accelerometers. DTIP: Design, Test, Integration and Packaging of MEMS/MOEMS, Apr 2008, Nice, France. pp.100-104, 10.1109/DTIP.2008.4752961. lirmm-00333632

\section{HAL Id: lirmm-00333632 https://hal-lirmm.ccsd.cnrs.fr/lirmm-00333632}

Submitted on 4 Sep 2019

HAL is a multi-disciplinary open access archive for the deposit and dissemination of scientific research documents, whether they are published or not. The documents may come from teaching and research institutions in France or abroad, or from public or private research centers.
L'archive ouverte pluridisciplinaire HAL, est destinée au dépôt et à la diffusion de documents scientifiques de niveau recherche, publiés ou non, émanant des établissements d'enseignement et de recherche français ou étrangers, des laboratoires publics ou privés. 


\title{
Study of first-order thermal $\Sigma \Delta$ architecture for convective accelerometers
}

\author{
Olivier LEMAN \\ Frédérick MAILLY \\ Laurent LATORRE \\ Pascal NOUET \\ Laboratoire d'Informatique, de Robotique et de Microélectronique de Montpellier (LIRMM) \\ UMR CNRS 5506 - University Montpellier II - France \\ Contact: mailly@lirmm.fr
}

\begin{abstract}
This paper presents the study of an original closed-loop conditioning approach for fully-integrated convective inertial sensors. The method is applied to an accelerometer manufactured on a standard CMOS technology using an auto-aligned bulk etching step. Using the thermal behavior of the sensor as a summing function, a first order sigma-delta modulator is built. This "electrophysical" modulator realizes an analog-to-digital conversion of the signal. Besides the feedback scheme should improve the sensor performance.
\end{abstract}

\section{INTRODUCTION}

Many studies report the design of open-loop convective accelerometers [1-10] but very few of them concern the conditioning electronics. Generally, a simple instrumentation amplifier is used. For long term stability and room temperature compensation, temperature control of the heater was studied by [6] and is implemented in a commercial sensor [4]. However, to our knowledge, no study deals with a real closed-loop architecture, where the effect of the acceleration on the detectors' temperature is compensated.

Sensor working principle is quite simple. The prototype realized at LIRMM (fig.1) using a standard CMOS technology features three polysilicon resistors embedded inside three suspended bridges. The central bridge is heated by Joule effect, and a thermal gradient is thus created in the surrounding air. The two lateral bridges are resistive temperature sensors with a Positive Temperature Coefficient (PTC). Without acceleration, both sensing bridges measure the same temperature due to symmetry reasons. Acceleration modifies the temperature profile due to free convection phenomenon along the sensitive axis which is perpendicular to the bridges. The temperature difference generated between the sensing bridges is converted into a differential output voltage by a Wheatstone bridge setup.

These sensors suffer from several weaknesses, such as:

- A quite low intrinsic sensitivity in the range of $1 \mathrm{mV} / \mathrm{g}$, which implies the design of a high-gain and low-noise amplifier.

- A non-linear acceleration-to-differential-temperature transfer function since the temperature difference between the sensors is limited by the temperature increase of the heater.
- A thermal inertia of the detectors which limits the bandwidth of the sensor to $50 \mathrm{~Hz}$. This is a technological limit. It was proven in previous studies that convective bandwidth higher than $100 \mathrm{~Hz}$ could be reached through a reduction of the cavity, making thermal inertia of detectors the limiting factor [9].

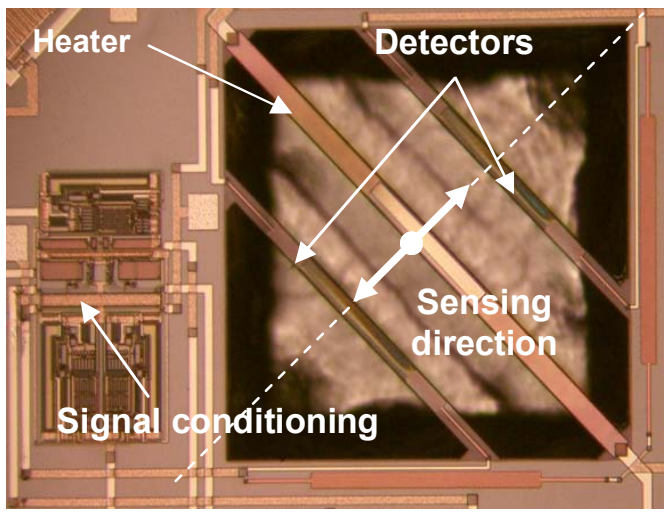

(a)

Fig. 1. Prototype micrograph

These limitations lead us to develop a closed-loop architecture in the form of an electro-physical sigma-delta modulator [11]. The sensor conditioner has a direct-digital output in the form of a bit-stream and compensates for the thermal inertia of the sensing bridges. The proposed feedback loop cancels the temperature difference between the two detectors by generating a compensation thermal power. This method uses Joule's effect, so that it can elevate the temperature of the "cold" detector, but the "hot" detector cannot be cooled this way. In order to solve this problem, the modulator slightly shifts the thermal bias point of the detectors by using a common-mode bias power which is then cancelled or increased depending on the desired action.

The first part deals with the modeling of the convective accelerometer, in order to realize behavioral simulations. In the second part we propose the electronic design of a convective accelerometer with thermal sigma-delta modulator conditioning. Finally, behavioral simulations of the proposed system are performed under the Matlab- 
Simulink environment and the results are discussed.

As a foreword, we want to stress the point that we have experimentally validated the thermal sigma-delta modulator principle and the proposed behavioral model [10, 12 and 13], giving strength to this design study.

\section{OPEN-LOOP SENSOR MODELING}

The study and modeling of the convective accelerometer sensing cell was published in previous studies [10, 13]. Using FEM simulations and characterization results, the model of fig. 2 was developed.

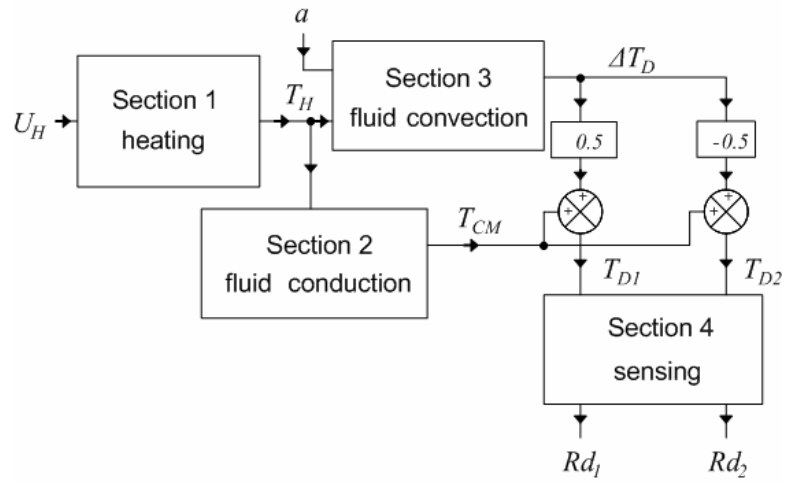

Fig. 2. Convective sensor model

Section 1 models the average temperature of the heating bridge:

$$
\Delta T_{H}=T_{H}-T_{A}=R_{t h} \times P_{H}
$$

Where $T_{H}$ and $T_{A}(\mathrm{~K})$ are respectively the average heater temperature and ambient temperature, $R_{t h}\left(\mathrm{~K} \mathrm{~W}^{-1}\right)$ is the equivalent thermal resistance of the beam and $P_{H}(\mathrm{~W})$ is the joule power dissipated in the heater resistance. Section 2 describes the conduction heat transfer, which is responsible for the common-mode temperature of the detectors. This model is based on a cylindrical geometry approximation and the common-mode temperature at a distance $r$ from the heater is ruled by expression 2 :

$$
T+\gamma \frac{T^{2}}{2}=\frac{\left[\left(T_{H}-T_{A}\right)+\frac{\gamma}{2}\left(T_{H}{ }^{2}-T_{A}^{2}\right)\right]}{\ln \left(\frac{r_{2}}{r_{1}}\right)} \cdot \ln \left(\frac{r}{r_{1}}\right)+T_{H}+\gamma \frac{T_{H}^{2}}{2}
$$

Section 3 expresses convective transfer from which depends the output signal. The temperature profile established in section 2 deforms, producing a temperature difference between the detectors $\Delta T_{D}$. This temperature difference has been found to be proportional to the Grashof number [1] for $\Delta T_{D}<<\left(T_{H}-T_{A}\right)$ :

$$
\Delta T_{D}=\frac{S . G r}{1+\tau p}=S \cdot \frac{a \beta \rho^{2}\left(T_{H}-T_{A}\right) l^{3}}{\mu^{2}} \cdot \frac{1}{1+\tau p}
$$

with $S$ (in K) a fitting coefficient (extracted from FEM analysis and experimental results) which represents the system sensitivity, a $\left(\mathrm{m}_{\mathrm{s}} \mathrm{s}^{-2}\right)$ the acceleration, $\rho\left(\mathrm{kg} \cdot \mathrm{m}^{-3}\right)$ the gas density, $\beta\left(\mathrm{K}^{-1}\right)$ the gas coefficient of expansion, $\mu$ $\left(\mathrm{kg} \cdot \mathrm{m}^{-1} \cdot \mathrm{s}^{-1}\right)$ the gas viscosity, $l(\mathrm{~m})$ a linear dimension related to the cavity volume and geometry and $\tau$ (s) the time constant of the fluid ( $\tau=0.5 \ldots 12 \mathrm{~ms}$, depending on the cavity geometry and dimensions [9]).

Finally, section 4 implements detectors transfer function taking into account the temperature sensitivity of polysilicon $\left(T C R=9.10^{-4} / \mathrm{K}\right)$ and the thermal time constant of the detecting bridges $\left(\tau_{D}=3.3 \mathrm{~ms}\right)$ :

$$
R d_{i}=R d_{i, n o m}\left(1+T C R\left(T_{C M} \pm \frac{1}{2} \Delta T_{D}\right) \frac{1}{1+\tau_{D} p}\right)
$$

The resistance variations of the PTC sensors are converted into a differential output voltage by means of a Wheatstone bridge with a sensitivity $S_{\text {wheat }}(\mathrm{V} / \mathrm{K})$ :

$$
S_{\text {Wheat }}=\frac{V d d}{4} \frac{T C R}{1+T C R \cdot T_{M C}}
$$

The Johnson noise of the detectors is finally added to the model since it has been identified as the limiting factor for the sensor resolution. In our prototype, the heating power is $42 \mathrm{~mW}$, heater temperature is $720 \mathrm{~K}$ and detectors commonmode temperature is $423 \mathrm{~K}$. The convection transfer function can thus be linearized as $\Delta T_{D}=a \cdot K_{M E M S}$ with sensitivity $K_{M E M S}=1.53 \mathrm{~K} / \mathrm{g} . K_{M E M S}$ is an empirical, composite parameter including the parameters of expression 3 . Wheatstone bridge's Johnson noise $\left(4 k_{b} T_{C M} R\right)$ generated by the $50 \mathrm{k} \Omega$ resistors is $34 \mathrm{nV} / \sqrt{\mathrm{Hz}}$ over a $6 \mathrm{MHz}$ bandwidth limited by the $1 \mathrm{pF}$ comparator input capacitance. Accelerometer sensitivity is $1.55 \mathrm{mV} / \mathrm{g}$, and its intrinsic resolution is $98 \mu$ grms over the $1-20 \mathrm{~Hz}$ bandwidth.

In order to study the conditioning electronics, a small signal model of the sensor was derived (fig. 3). Such a simplified model is suitable for fast behavioral simulations under the Matlab-Simulink framework.

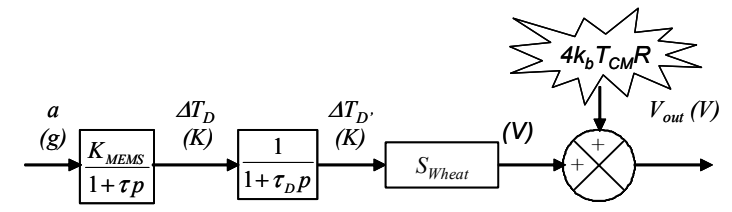

Fig. 3. Small-signal model of the sensor

This model was used in a previous study of open-loop amplifier architectures to show that a Wheatstone bridge bias modulation together with a synchronous detection scheme would allow to cancel the low-frequency noise of a standard CMOS amplifier and to reach resolutions close to the intrinsic performance of the convective accelerometer cell [12].

\section{ClOSED-LOOP SENSOR ARCHITECTURE}

The convective sensing element studied here is an evolution of the previous version presented in part 2. Two temperature detectors are added in the suspended bridges in 
order to create a Wheatstone bridge with 4 sensitive resistors (fig. 4-a). The resulting sensor features twice the original $S_{\text {Wheat }}$ sensitivity and then twice the previous intrinsic signalto-noise ratio. Considering the operating conditions stated in part 2 , the sensitivity reaches now $3.1 \mathrm{mV} / \mathrm{g}$; the intrinsic noise of $11 \mu \mathrm{g} / \sqrt{\mathrm{Hz}}$ leads to a $49 \mu \mathrm{grms}$ resolution over the 1 $20 \mathrm{~Hz}$ bandwidth.

In order to allow a thermal feedback of the suspended bridges, self-heating of the temperature detectors due to the biasing of the Wheatstone bridge is now taken into account in the model, resulting in a slight shift of the common mode temperature of the two suspended bridges. Moreover, we noticed that this self-heating interestingly falls in the same range as the power necessary to compensate for the convection phenomenon. The thermal feedback is then simply achieved by shorting the sensitive resistors using MOS switches (fig. 4-a). Table 1 gives the power dissipation in the two suspended bridges as a function of the MOS switches command. When both feedback commands are low, the Wheatstone bridge is regularly biased, and the power dissipation is $P_{0}=V^{2} /\left(2 R\left(T_{M C}\right)\right)=225 \mu \mathrm{W}$ in each suspended bridge. When feedback_1 is high, resistors in bridge 2 are shorted and resistors in bridge 1 are biased at $V_{d d}$. Power dissipation is then null in bridge 2 and $P_{\max }=2 V^{2} /\left(R\left(T_{M C}\right)\right)=$ $900 \mu \mathrm{W}$ in bridge 1 . Feedback_2 activation generates a complementary power dissipation effect. These two feedback paths are used to compensate the temperature difference generated by convection (i.e. by the acceleration).

TABLE 1

Control signals and corresponding power dissipation in sensing bridges

\begin{tabular}{|c|c|c|c|}
\hline \multicolumn{2}{|c|}{ command } & \multicolumn{2}{c|}{ dissipation } \\
\hline feedback_1 & feedback_2 & bridge 1 & bridge 2 \\
\hline 0 & 0 & $\mathrm{P}_{0}$ & $\mathrm{P}_{0}$ \\
\hline 1 & 0 & $\mathrm{P}_{\max }$ & 0 \\
\hline 0 & 1 & 0 & $\mathrm{P}_{\max }$ \\
\hline
\end{tabular}

This principle requires a 2-phase time scheduling and fig. 4.b. presents the corresponding chronograms:

- $\quad$ phase $\Phi 1$, the sensor is in reading mode. Both feedback paths are disabled, and the Wheatstone bridge under regular bias delivers the error signal $\mathrm{V}_{\text {out }}$. If $\mathrm{V}_{\text {out }}$ is negative, it means that the two detectors embedded on bridge 2 are the warmer and then the comparator output is low. If it is positive, the two detectors in bridge 1 are the warmer and the comparator output is high.

- $\quad$ phase $\Phi 2$, the sensor is in the feedback mode, thus one of the two feedback paths is enabled according to the comparator output. For example, if the comparator output is low, feedback 1 is enabled: power dissipation is then null in bridge 2 and equal to $P_{\max }$ in bridge 1 . During this phase, the Wheatstone bridge output is an invalid $+/-V_{d d}$.

The longer $T_{\text {comp }}$ is, the higher compensation power $P_{\text {comp }}$ is generated, according to the $P_{\text {comp }}=\alpha P_{\max }$ relationship. Sensor full-scale is adjusted through the duty-cycle $\alpha$ of feedback pulses. A null acceleration entails that each bridge is heated by an average $P_{\text {comp }} / 2$ power which slightly shifts the thermal common-mode of the detecting bridges.

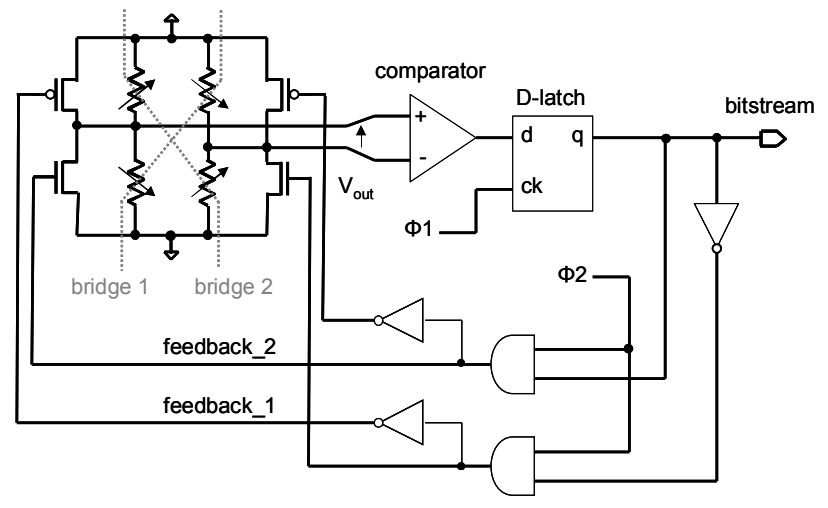

(a)

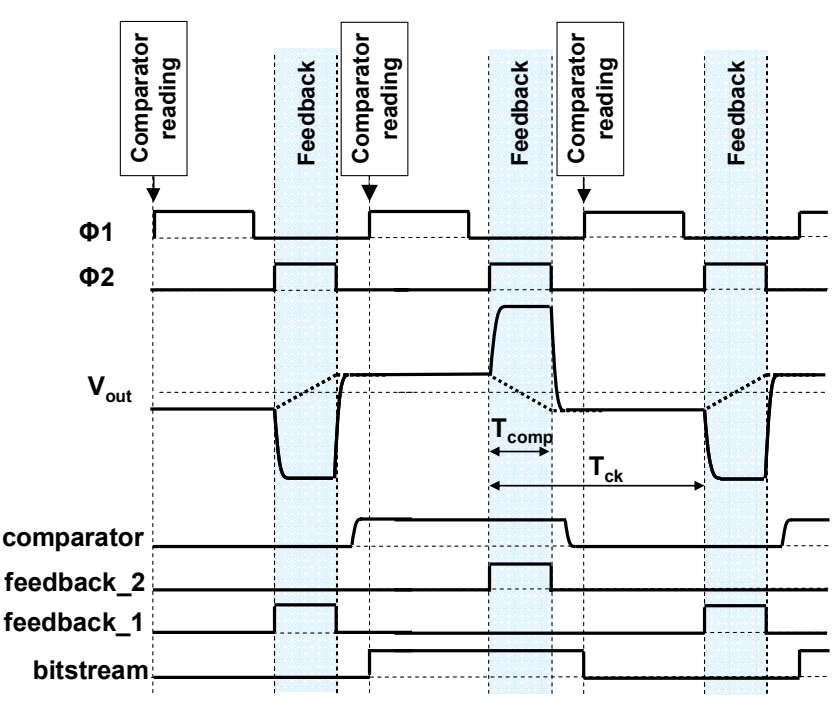

(b)

Fig. 4. Modified sensor architecture (a) and chronograms (b)

\section{CLOSED-LOOP SENSOR SIMULATION}

First the small signal model of the sensor (fig.3) is modified to include the power-feedback scheme of the sigma-delta modulator (fig.5). This is achieved by converting the temperature variations of the bridges into thermal power using the characterized thermal resistance of the detecting bridges, $R_{t h}=10^{4} \mathrm{~K} / \mathrm{W}$. Consequently, system input becomes a thermal power $\Delta P_{D}$ resulting from acceleration:

$\Delta P_{D}=\frac{K_{M E M S}}{R_{t h}} \frac{a}{1+\tau p}$

In this first order thermal sigma-delta modulator, the electro-thermal response of the detecting bridges replaces the integrator. This thermal filter is a first-order low-pass with a cut-off frequency of $48 \mathrm{~Hz}$, so that it has a DC gain of $10^{4} \mathrm{~K} / \mathrm{W}$ in the $\mathrm{DC}-48 \mathrm{~Hz}$ part of the spectrum, thus acting as a leaking integrator. 


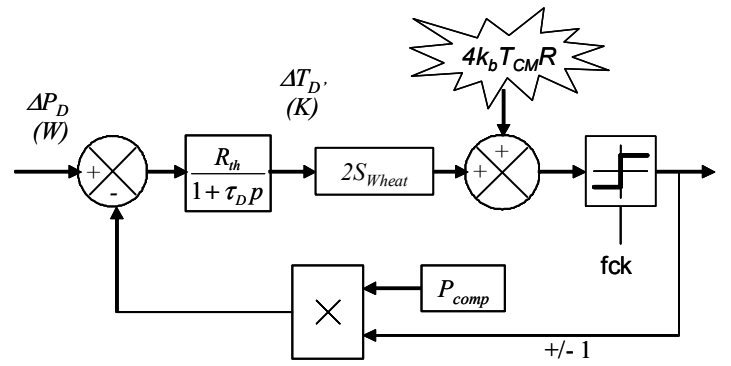

Fig.5. Sigma-delta modulator simulation model

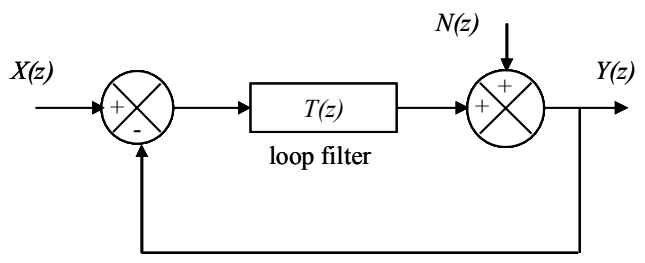

Fig. 6. Linearized model of the sigma-delta modulator

In order to study the impact of this non-ideal integrator, we use a linearized model of the modulator (fig. 6) where $\mathrm{T}(\mathrm{z})$ is the $\mathrm{z}$-transform of the loop filter transfer function and $\mathrm{N}(\mathrm{z})$ is the quantization noise, under the white-noise assumption. The Johnson noise is not considered in this model.

Quantization noise transfer function $\mathrm{Y}(\mathrm{z}) / \mathrm{N}(\mathrm{z})$ is :

$$
\operatorname{NTF}(z)=\frac{1}{1+T(z)}
$$

Considering an ideal integrator as the loop filter, the NTF equals $1-z^{-1}$. Hence, quantization noise is shaped over the full spectrum. Now if we consider the thermal filter which transfer function in the $\mathrm{z}$-domain writes:

$$
T(z)=\frac{z^{-1}}{1-\alpha \cdot z^{-1}}
$$

with $\alpha=e^{-\frac{T e}{\tau_{D}}}$ close to 1 , the NTF becomes $1-\alpha \cdot z^{-1}$ [14]. This NTF becomes flat in the DC- $48 \mathrm{~Hz}$ part of the spectrum, meaning that the quantization noise is unshaped in this part of the spectrum (fig. 7-b).

Transient behavioral simulations of the system were carried-out in Matlab-Simulink. We studied the performance of the architecture using a spectral analysis of the output bitstream (fig. 8).

If we compare results of figures 7 and 8 , we notice that the behavior of the simulation model is close to the prediction of the analytical model in the low-frequency spectrum, the lowpass thermal loop filter leads to a flat quantization noise from 1 to $48 \mathrm{~Hz}$ (fig.8-a). Resolution is $1.48 \mathrm{mg}$ over the 1 $20 \mathrm{~Hz}$ bandwidth, whereas the same modulator with an ideal integrator (fig.8-b) would have a $0.26 \mathrm{mg}$ resolution. Moreover, a slight distortion appears. This phenomenon is common for first-order modulators, it corresponds to the oril 2008

third harmonic and its amplitude is below $0.1 \%$ of the main peak.

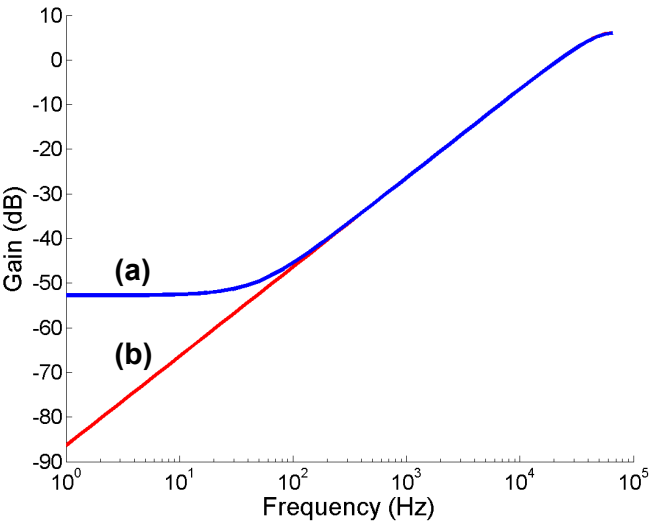

Fig. 7. Noise transfer functions for an ideal integrator (b) and for the thermal integrator (a) $@ f_{c k}=131 \mathrm{kHz}$.

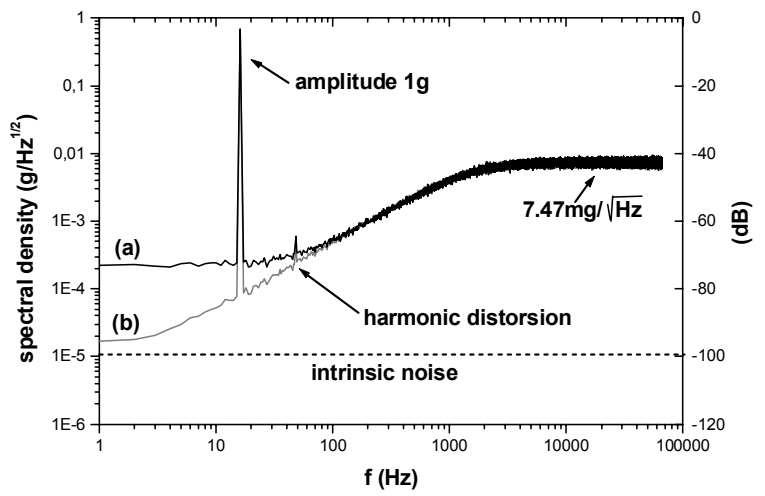

Fig. 8. FFT of the bit-stream for the sigma-delta modulator model (a), same modulator with an ideal integrator (b), $f_{c k}=131 \mathrm{kHz}, \pm 2 \mathrm{~g}$ full-scale, input : $16 \mathrm{~Hz}$ sinusoidal acceleration, amplitude $1 \mathrm{~g}$

However, figure 8 shows that quantization noise is unshaped in the upper part of the spectrum. Above $1.6 \mathrm{kHz}$, the power of the error signal falls below the Johnson noise floor of the Wheatstone bridge. This results in a random output of the 1-bit quantizer. In the end, oversampling simply leads to a quantization noise spreading. In absence of shaping from $1.6 \mathrm{kHz}$ to $f_{c k} / 2$ (e.g. about $98 \%$ of the spectrum), more than $99 \%$ of the quantization noise power is unshaped. We can have a good estimation of the quantization noise spectral density in the $1.6 \mathrm{kHz}-\left(f_{c k} / 2\right)$ spectrum, neglecting the $48 \mathrm{~Hz}-1.6 \mathrm{kHz}$ first order noise shaping. Assuming that the total bit-stream power is constant, the quantization noise should be given by:

$$
\frac{\sqrt{S_{b}^{2}-S_{a}^{2}}}{\sqrt{f_{c k} / 2}}=7.31 \mathrm{mg} / \sqrt{H z}
$$

where $S_{b}=2 \mathrm{~g}_{\mathrm{RMS}}$ is the bit-stream signal power, and $S_{a}=$ 
$1 / \sqrt{2} \mathrm{~g}_{\mathrm{RMS}}$ is the acceleration signal.

Simulations of the figure 8 give $7.47 \mathrm{mg} / \sqrt{ } \mathrm{Hz}$. This proves that the $48 \mathrm{~Hz}-1.6 \mathrm{kHz} 1 \mathrm{st}$ order shaping has little effect on the overall quantization noise shaping.

Figure 9 presents the signal transfer functions of the two modulators. As a consequence of the finite DC gain of the thermal, the DC gain of the electro-thermal modulator is $0.3 \mathrm{~dB}$ lower than the theoretical gain. Their $-3 \mathrm{~dB}$ cut-off frequencies are both about $1.6 \mathrm{kHz}$. Above this frequency it was shown that the Johnson noise of the Wheatstone bridge disturbs the modulator operating. It's likely that this phenomenon limits also the modulator bandwidth.

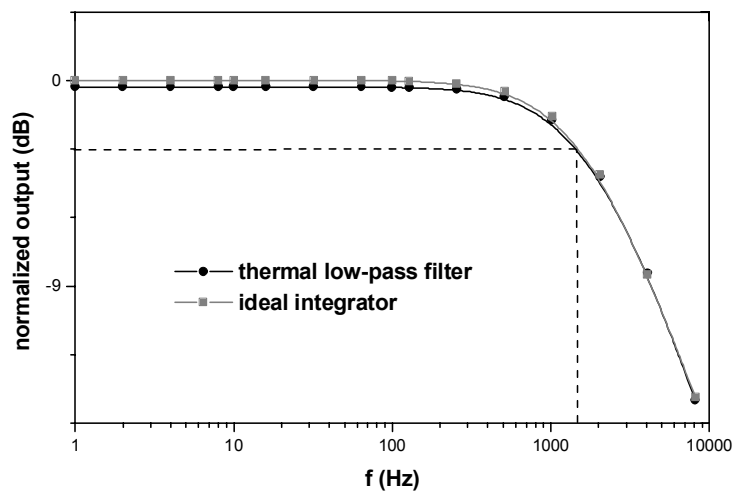

Fig. 9. Signal transfer functions of the modulators, $f_{c k}=131 \mathrm{kHz}, \pm 2 \mathrm{~g}$ fullscale

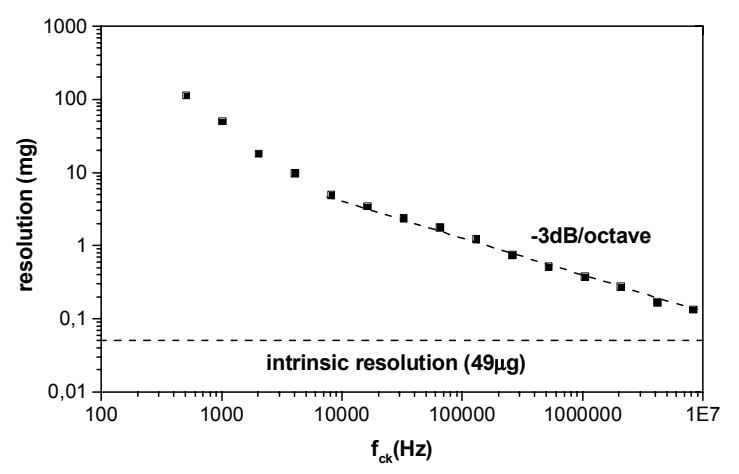

Fig. 10. Resolution $(\mathrm{BW}=1-20 \mathrm{~Hz})$ versus modulator clock frequency

Finally, the same system was simulated for various clock frequencies $f_{c k}$ and the corresponding resolutions were computed over the bandwidth $1-20 \mathrm{~Hz}$. The results, plotted on figure 10 , show that for clock frequencies higher than 10 $\mathrm{kHz}$, the signal-to-noise ratio increases at a $3 \mathrm{~dB} /$ octave rate. This poor improvement comes from the quantization noise spreading due to the absence of quantization noise shaping above $1.6 \mathrm{kHz}$.

A $132 \mu \mathrm{g}$ resolution was simulated with a $8.4 \mathrm{MHz}$ clock frequency. Higher clock frequencies resulted in bitstreams too large to be analyzed with Matlab. From the $-3 \mathrm{~dB} /$ octave SNR increase, a modulator with a clock frequency of about $100 \mathrm{MHz}$ would reach the intrinsic resolution of the convective sensor; say $49 \mu \mathrm{g}$ over the $1-20 \mathrm{~Hz}$ bandwidth.

This result suggests the design of a second order sigma- delta modulator architecture, expecting a lower distortion and lower frequency clocks through a better noise shaping.

\section{CONCLUSION}

This study reports an original smart-sensor architecture for thermal convective accelerometers based on the thermal sigma-delta modulator principle. We consider a first order sigma-delta architecture. Using the side effects of self heating and thermal inertia of detectors, most of the analog signal processing is performed in the thermal domain, drastically reducing the amount of analog electronics. Simulation results show that a high modulator clock frequency is required to reach the intrinsic noise of the sensor, motivating the design of a higher order modulator.

\section{REFERENCES}

[1] A.M. Leung, J. Jones, E. Czyzewska, J. Chen and B. Woods, "Micromachined accelerometer based on convection heat transfer", IEEE MEMS'98, Heidelberg, Germany; 25-29 Jan. 1998, pp. 627630

[2] V. Milanovic, E. Bowen, N. Tea, J. Suehle, B. Payne, M. Zaghloul, and M. Gaitan, "Convection-based Accelerometer and Tilt Sensor Implemented in Standard CMOS", International Mechanical Engineering and Exposition, MEMS Symposia, Anaheim, CA, (1998), pp. 487-490

[3] S. Billat, H. Glosch, M. Kunze, F. Hedrich, J. Frech, J. Auber, H. Sandmaier, W. Wimmer and W. Lang, "Micromachined inclinometer with high sensitivity and very good stability", Sensors and Actuators A 97-98 (2002), pp. 125-130

[4] www.memsic.com

[5] X.B. Luo, Y.J. Yang, F. Zheng, Z.X. Li and Z.Y. Guo, "An optimized micromachined convective accelerometer with no proof mass", J. Michromech. Microeng. 11 (2001), pp. 504-508.

[6] G. Kaltsas, D. Goustouridis and A.G. Nassiopoulou, "A thermal convective accelerometer system based on a silicon sensor - Study and packaging", Sensors and Actuators A 132 (2006) pp. 147-153.

[7] R. Zhu, H. Ding, Y. Su and Z. Zhou, "Micromachined gas inertial sensor based on convection heat transfer", Sens. Actuators A 130131 (2006), pp. $6-74$

[8] F. Mailly, A. Giani, A. Martinez, R. Bonnot, P. Temple-Boyer and A. Boyer, "Micromachined thermal accelerometer", Sensors and Actuators A: Physical, Volume 103, Issue 3, 15 February 2003, pp. 359-363

[9] A. Chaehoi, F. Mailly, L. Latorre and P. Nouet, "Experimental and finite-element study of convective accelerometer on CMOS", Sensors and Actuators A: Physical, Volume 132, Issue 1, November 2006, pp. 78-84

[10] O. Leman, F. Mailly, L. Latorre and P. Nouet, "HDL modeling of convective accelerometers for system design and optimization", Sensors and Actuators A: Physical, Volume 142, Issue 1, 10 March 2008, Pp. 178-184

[11] K.A.A. Makinwa, V. Székely, J.H. Huijsing, "Modeling and simulation of thermal sigma-delta modulators", Instrumentation and Measurement Technology Conference, Anchorage, AK, USA, 2123 May 2002, pp. 261-264

[12] O. Leman, A. Chaehoi, F. Mailly, L.Latorre and P. Nouet, "A comparative study of conditioning architectures for convective accelerometers", IEEE Sensors Conference, Atlanta, GA, USA, 2831 October 2007

[13] O. Leman, A. Chaehoi, F. Mailly, L. Latorre and P. Nouet, "Modeling and system-level simulation of a CMOS convective accelerometer", Solid-State Electronics, Volume 51, Issues 1112, November-December 2007, pp. 1609-1617

[14] P.M. Aziz, H.V. Sorensen, J.V. der Spiegel, "An overview of Sigma-Delta Converters", IEEE Signal Processing Magazine, 10535888, January 1996 